\title{
Method for Traffic Flow Estimation using On- dashboard Camera Image
}

\author{
Kohei Arai \\ Graduate School of Science and Engineering \\ Saga University \\ Saga, Japan
}

\author{
Steven Ray Sentinuwo \\ Department of Electrical Engineering \\ Sam Ratulangi University \\ Manado, Indonesia
}

\begin{abstract}
This paper presents the method to estimate the traffic flow on the urban roadway by using car's on-dashboard camera image. The system described, shows something new which utilizes only road traffic photo images to get the information about urban roadway traffic flow automatically.
\end{abstract}

Keywords-traffic flow estimation; on-dashboard camera; computer vision.

\section{INTRODUCTION}

Due to the increasing of the traffic densities nowadays, there exists a growing demand for advanced systems that can provide the essential traffic and travel information for the drivers to improve the traffic quality and travel optimization. The comprehensive and accurate of traffic information is the important aspect in order to manage the roadway network and to provide the navigation service for the road users. The density value measurement is one of the important aspect of transportation management. Intelligent Transportation System is a breakthrough technology that combines components of information management systems to create better transportation system. Currently, demands on the system are growing rapidly with an estimated travel demand increase of $30 \%$ over the next ten years[1]. The efficient of transportation system can be an alternative to increase the roadway capacity in order to prevent the traffic congestion at current levels from getting worse.

Vehicle detection and surveillance technologies are an integral part of ITS, since they gather all or part of the data that is used in ITS. There is an estimation that an investment in ITS will allow for fewer miles of road to be built, thus reducing the cost of mitigating recurring congestion by approximately 35 percent nationwide. New vehicle detection and surveillance technologies are constantly being developed and existing technologies improved, to provide speed monitoring, traffic counting, presence detection, headway measurement, vehicle classification, and weigh-in-motion data.

There are many methods and technologies for traffic measurement. However, the conventional measurement methods, such as "in-situ" technologies which are measure traffic data by locating detectors or sensors along the roadway, have some drawbacks. The use of these conventional methods for collecting data is necessary but not sufficient because of their limited coverage area and expensive costs of implementation and maintenance. Then as the alternative to the conventional methods there are alternative methods, such as based on the vehicle location or Floating Car Data method (FCD), and collecting data from "in-vehicle" devices.

This paper proposes a method for traffic measurement using the approach of collecting data from "in-vehicle" device which is by utilizing camera device that located on the car`s dashboard. The image that captured by digital camera photo then was analyzed to get the estimation value of roadway traffic flow condition. A traffic measurement using digital camera device can be a practical and low cost solution compare to the conventional traffic measurement methods.

This paper is organized as follow. The brief description about traffic flow measurement methods and procedures is quick review in Section 2. Section 3 described some previous research that taking into account traffic monitoring models. In Section 4, the proposed method of traffic flow measurement using on-dashboard camera image is explained. Section 5 and Section 6 show the results of simulation study and experiment in the real traffic condition, respectively. Finally, in Section 7, we present the conclusion and the future works.

\section{TRAFFIC FlOW MEASUREMENT}

Researchers in the area of Intelligent Transportation System have been greatly interested in the various of traffic management and monitoring applications. For several years, under growing pressure for improving traffic management, a wide variety of applications have been developed. Some researchers focus on the theoretical aspect that involves driver behavior into the transportation model [2][3][4][5]. While the other focus on the development of application and tools of intelligent transportation system.

There are some items of interest in traffic theory, have been the following[6]:

- Rates of flow, which evaluates the number of vehicles per unit of time;

- Speeds, which evaluates the distance per unit time;

- Travel time over a known length of road, or sometimes refer to the invers of speed;

- Occupancy, that is percent of time a point on the road is occupied by vehicles;

- Density, which is refer to the number of vehicles per unit distance.

- Time headway between vehicles (time per vehicle); 
- Spacing, or space headway between vehicles (distance per vehicles);

- Concentration, that is measured by density of occupancy.

The capability of traffic measurement to gather transportation data have changed over the nearly sixty year span of interest in traffic flow measurement. And to be more growing which there have been a large number of freeway and number of population. Indeed, traffic measurement methods are still changing, there are five common procedures in this area:

- Measurement at a point;

- Measurement over a short section, by which meant less than about 10 meters $(\mathrm{m})$;

- Measurement over a length of road, usually at least 0.5 kilometers $(\mathrm{km})$;

- Using moving observer along the traffic stream;

- Wide-area samples obtained simultaneously from a number of vehicles, as part as the Intelligent Transportation System (ITS).

In moving observer method, there are two common approaches[6]. The first one is the simple floating car data procedure (FCD). This approach intends to record speeds and travel times as the function of time and location along the road. The intention of this approach is the floating car as the observer car behaves as an average vehicle within the traffic stream. However, this approach cannot give provide average speed data. This one just effective for producing a qualitative information about roadway conditions and operations. One form of this approach uses a person in the floating car to record speeds and travel times. The second form uses a modified recording speedometer of the type regularly used in longdistance trucks or buses. While the drawback of this approach is that it means there are usually significantly fewer speed observations than volume observations.

The second one introduced by Wardrop et.al.[7] for urban traffic measurements. This approach intends to obtain both speed and volume measurements simultaneously. This method is based on an observation vehicle that travels in both direction on the roadway. The formulae allow one to estimate both speeds and flows for one direction of travel. The formulae are as follow :

$$
\begin{aligned}
\mathrm{q} & =\frac{(x+y)}{\left(t_{a}+t_{w}\right)} \\
\bar{t} & =t_{w}-\frac{y}{q}
\end{aligned}
$$

where,

- $\mathrm{q}$, is the estimated flow on the road in the direction of interest,

- $\mathrm{x}$, is the number of vehicles traveling in the direction of interest, which are met by the observation car while traveling in the opposite direction,
- $y$, is the net number of cars that overtake the observation car while traveling in the direction of interest,

- ta, is the travel time taken for the trip against the stream,

- tw, is the travel time for the trip with the stream,

- $\bar{t}$, is the estimate of mean travel time in the direction of interest.

In 1973, Wright revised the theory behind this method[8]. His paper finds that the method gives biased results, although the degree of bias is not significant in practice, and can be overcome. His paper proposed that the driver should fix the journey time in advance then stops along the way would not matter. He found also that the turning traffic (exiting or entering) can upset the calculation done using this method. A suggestion also said that a large number of observations are required for reliable estimation of speeds and flow rates in order to get the precision value.

\section{ROAD TRAFFIC MONITORING MODELS}

Most of the road traffic monitoring system are based on motion detection to make a segmentation to the region of the image. Zhu et.al[9]. presented VISATRAM, a system for automatic traffic monitoring using 2D spatio-temporal images. A TV cam- era is mounted above a highway to monitor the traffic through two slice windows, and a panoramic view image and an epipolar plane image are formed for each lane. If the regions contain the appropriate characteristics, vehicles are considered and can be counted or tracked as desired. Out of the motion detection techniques defined so far, the two most frequently used in road-traffic monitoring are the image difference method and the motion detection technique based on features.

The other system uses image difference technique[10]. This technique based on the fact that the differences between two frames captured at different time instants reveal regions in motion. An image difference $I$ is generated by calculating the absolute difference between two frames $\left(I_{1}\right.$ and $\left.I_{2}\right)$ and thresholding the result.

$$
I(x, y)= \begin{cases}0, & \text { if }\left|I_{1}(x, y)-I_{2}(x, y)\right| \leqslant \theta \\ 1, & \text { otherwise }\end{cases}
$$

where $\theta$ is an appropriate threshold. In the case of traffic monitoring, it is usual for $I_{1}$ to be the input frame and $I_{2}$ to be the reference frame (background). The reference frame is an image from the scene, without cars. The purpose of the threshold is to reduce the effects of noise and changes in the scene's lighting. The latter is a great problem in computer vision and it is usually necessary to use methods of dynamic updating of the reference frame to adapt to the scene's lighting.

Some complete traffic monitoring systems are available to date. The system that called TRIP (Traffic Research using Image Processing)[11] is designed to count vehicles traveling in a two-lane, two- way highway. The camera is placed in the highway, looking vertically onto the scene. 
By using a reference image, the input images are differentiated and the result is thresholded. This leads to a binary image where vehicles in motion should appear.

The greatest effort in the development of ITS was probably made by the U.S. Department of Transportation when it requested Mitretek Systems, Inc.[1] to carry out several ITS projects. The ones that stand out are those meant for a metropolitan ITS infrastructure, to which the following systems belong: Arterial Roads Control System, Highway Control System, Transit Control System (meant for public transportation in cities), Incident Control System, Emergency Control System, Electronic Collection System for Toll Roads (freeways), Electronic Fares Payment System (in-cities), Intersections between Freeways and Railroad Tracks, Regional Information for travelers and Integrated Systems. The subject continues to arouse a lot of interest, as can be seen in the article on vehicle detection and classification[12], where a six-stage system is proposed: (1) segmentation, (2) region tracking, (3) recovery of vehicle parameters, (4) vehicle identification, (5) vehicle tracking and (6) vehicle classification.

\section{TRAFFIC FLOW ESTIMATION USING ON-DASHBOARD CAMERA IMAGE}

As stated before, the purpose of this paper is to present a visual system which estimate the traffic flow by utilize digital camera which located on the car's dashboard to capture the road scenery image. Figure 1 shows the schematic diagram of the concept.

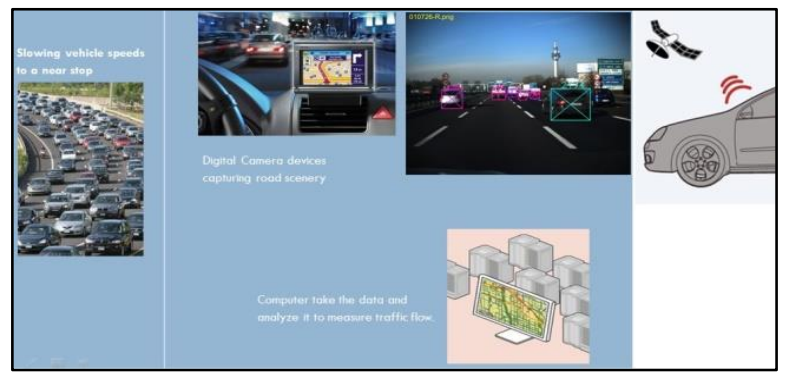

Figure. 1. Schematic diagram of the concept

This system uses a camera mounted on the car's dashboard which capture real traffic image.

\section{REAL TRAFFIC EXPERIMENT}

Empirical data is used in this experiment. Figure 2 shows the location of this experiment. The on-dashboard camera then capture the front scenery information. In this example, which is showed by Figure 3, we captured the real traffic situation and investigated for the opposite lane direction of the observer car.

This system uses four sub-step to identified the car. In the initial step, this system crop the raw image to get only the opposite direction lane image. This preprocessed image is showed by Figure 3. In the second step, the preprocessed image is gray-scaled. After that the threshold is applied to the image. Then the car is detected by using blob detection and some morphology step.

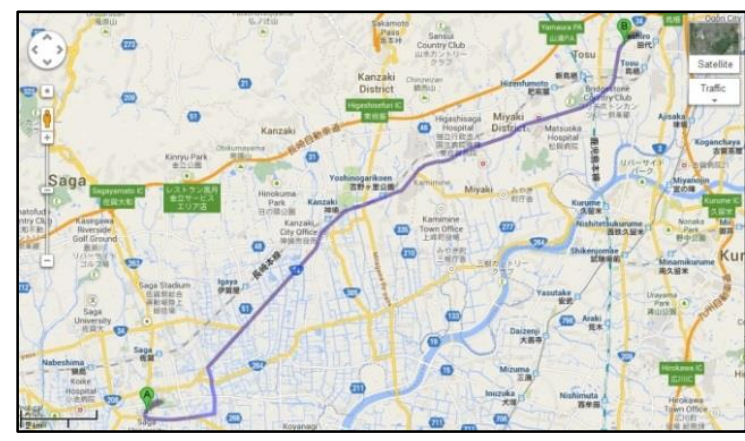

Figure. 2. The Location of Observation

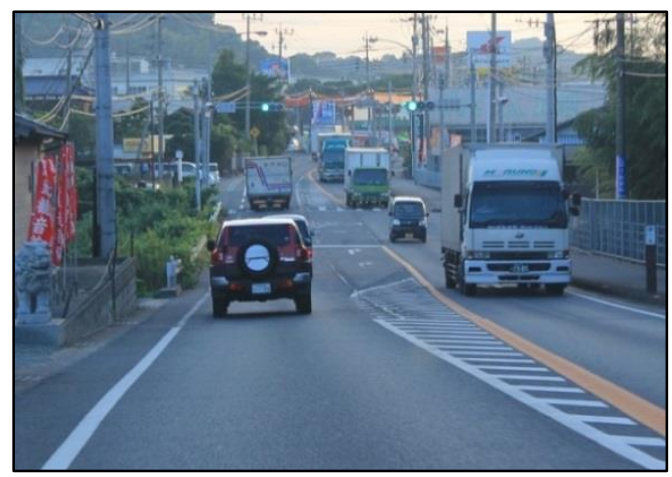

Figure. 3. Road traffic monitoring image

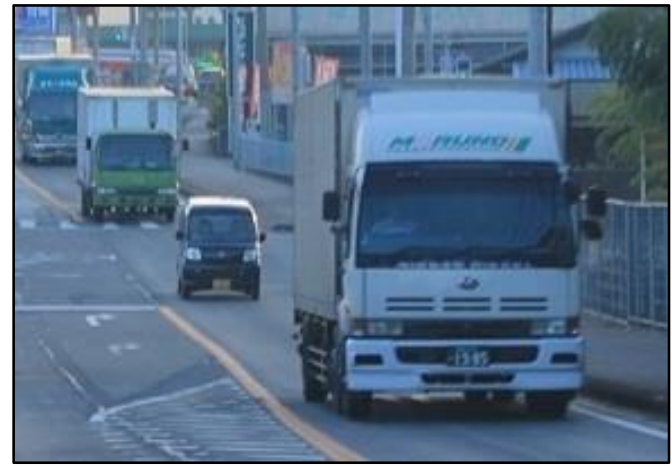

Figure. 4. Preprocessed Image

In the Figure 6, the difference matrix is applied to a threshold. The gray levels greater and lower than the threshold is updated as 1 and 0 , respectively. Which leads the car objects to be represented as black pixels.

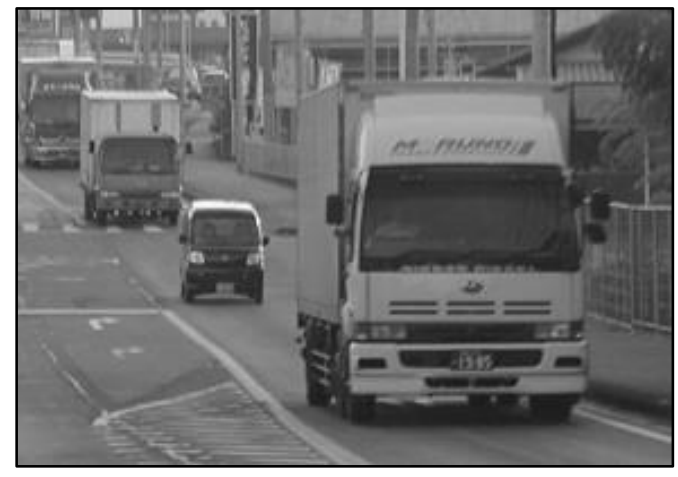

Figure. 5. Real Image in gray scales 


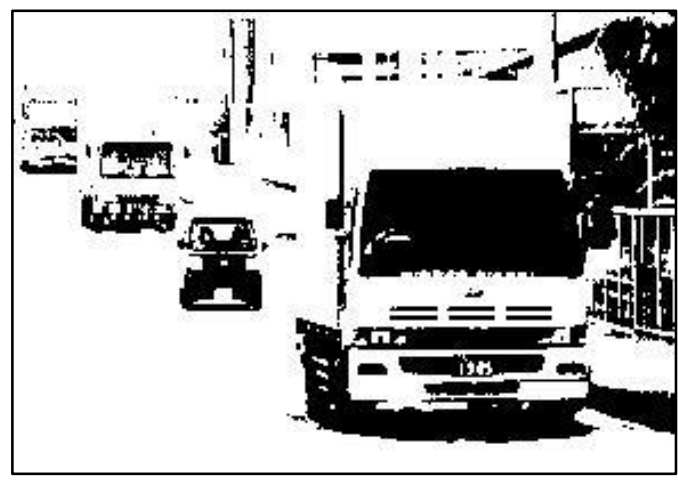

Figure. 6. Segmented Image

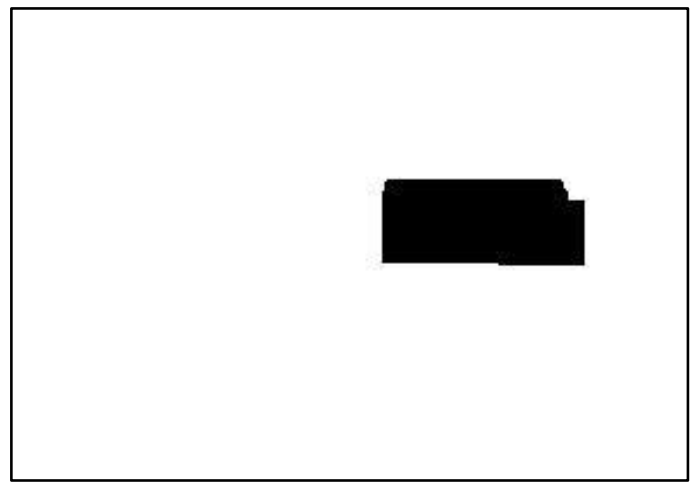

Figure. 7. Processed Image

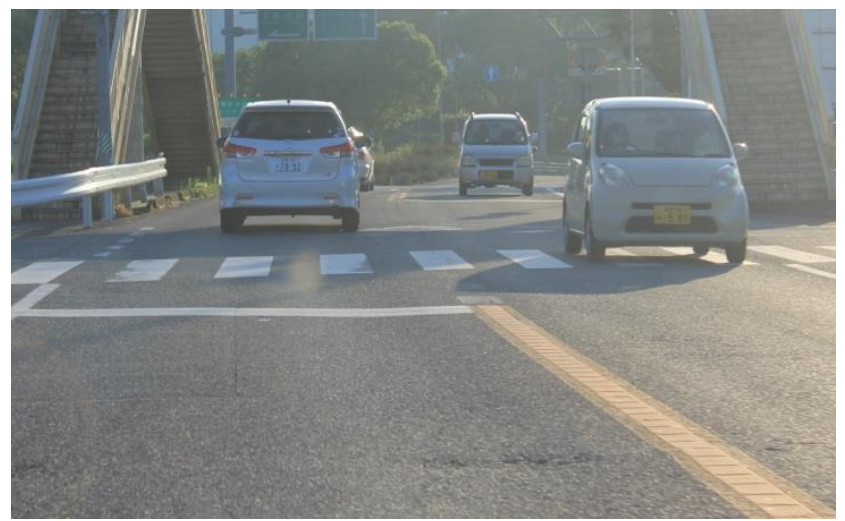

(a)Original

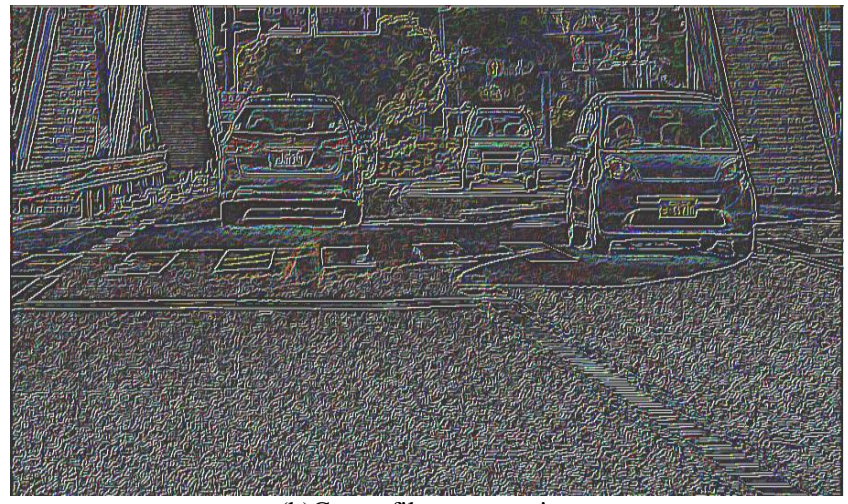

(b)Canny filter summation

Figure 8. Example of images of which it is hard to count the number of cars in front of the car in concern
As shown in Figure 8 (a) and (b), it is hard to count the number of cars in front of the car in concern because some cars are overlapped just a behind of the car just in front of the car in concern. It, however, the hidden cars (occluded cars) are appeared when they are observed in curved roads or in sloped roads as shown in Figure 9 (a) and (b). Where the image (a) shows the original image while the image (b) shows Canny filter with summation is applied for segmentations.
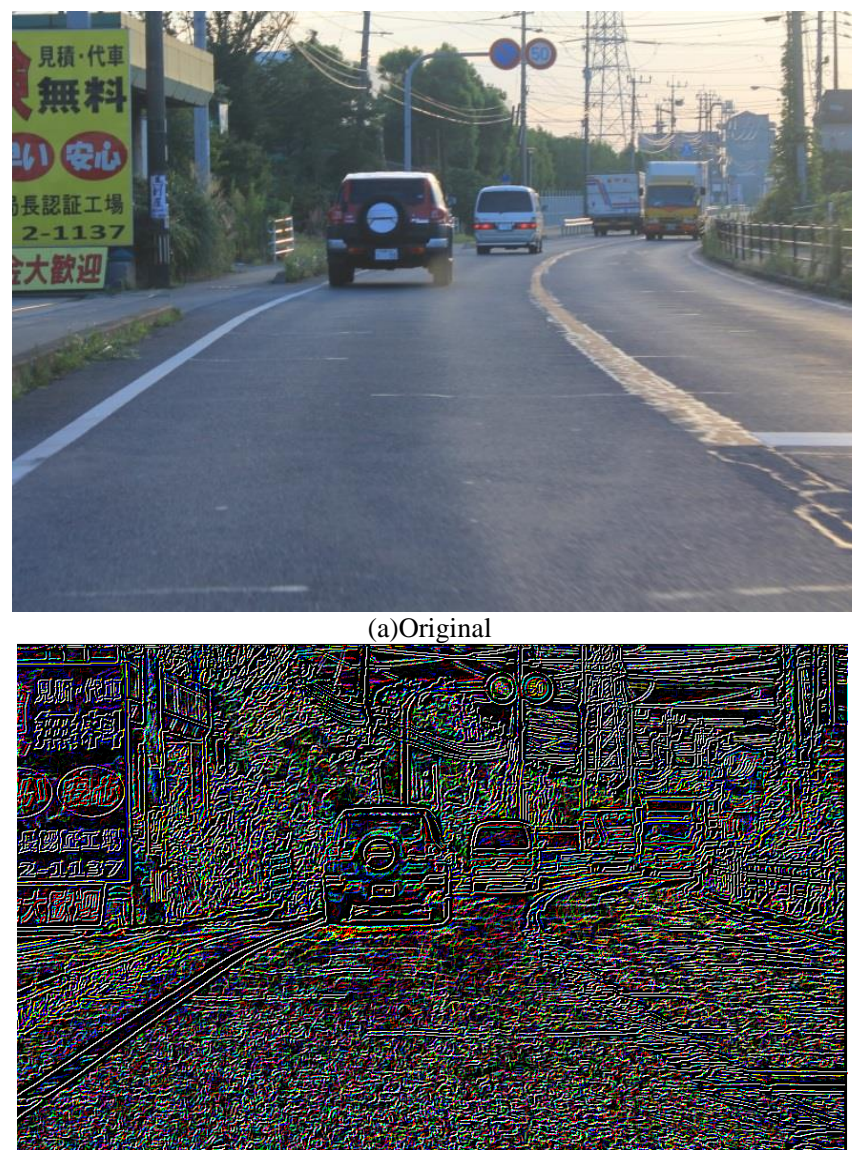

(b)Canny filter summation

Figure 9. Example of images of which it is possible to count the number of cars in front of the car in concern

\section{CONCLUSION AND FUTURE WORKS}

Automatic traffic flow estimation through on-dashboard camera is the new approach for the intelligent transportation system domain. This paper proposes a method for traffic measurement using the approach of collecting data from "invehicle" device which is by utilizing camera device that located on the car's dashboard. The image that captured by digital camera photo then was analyzed to get the estimation value of roadway traffic flow condition. A traffic measurement using digital camera device can be a practical and low cost solution compare to the conventional traffic measurement methods.

The future step needs the effective solution on how to remove the noise background and shadow that occur on the real traffic image. 


\section{REFERENCES}

[1] T. Proper, A, "Intelligent transportation system benefits," US Dep. Transp. Washingt. DC.

[2] A. Schadschneider, "The Nagel-Schreckenberg model revised," Eur. Phys. J. B, vol. 10, no. 3, pp. 573-582, 1999.

[3] K. Arai and S. R. Sentinuwo, "Effect of Driver Scope Awareness in the Lane Changing Maneuvers Using Cellular Automaton Model," Int. J. Adv. Res. Artif. Intell., vol. 2, no. 7, pp. 6-12, 2013.

[4] K. Arai and S. R. Sentinuwo, "Validity of Spontaneous Braking and Lane Changing with Scope of Awareness by Using Measured Traffic Flow," Int. J. Adv. Res. Artif. Intell., vol. 2, no. 7, pp. 13-17, 2013.

[5] K. Arai and S. R. Sentinuwo, "Validity of Spontaneous Braking and Lane Changing with Scope of Awareness by Using Measured Traffic Flow," Int. J. Adv. Res. Artif. Intell., vol. 2, no. 7, pp. 13-17, 2013.

[6] F. Hall, "Traffic stream characteristics," Washington, DC, 1975.

[7] J. . Wardrop and G. Charlesworth, "A Method of Estimating Speed and Flow of Traffic from a Moving Vehicle," in Proceedings of the Institution of Civil Engineers, Part II, Vol. 3, 1954, pp. 158-171.

[8] C. Wright, "A Theretical Analysis of the Moving Observer Method," Transp. Res. 7, pp. 293-311, 1973.

[9] Z. Zhu, G. Xu, B. Yang, D. Shi, and X. Lin, "VISATRAM: A real-time vision system for automatic traffic monitoring," Image Vis. Comput., 2000.

[10] M. Sonka, V. Hlavac, and R. Boyle, Image processing, analysis, and machine vision. Chapman \& Hall, 1999.
[11] K. Dickinson and C. Wan, "Road traffic monitoring using the TRIP II system," Road Traffic Monit. 1989

[12] S. Gupte and O. Masoud, "Detection and classification of vehicles," Intell. Transp. Syst., vol. 3, no. 1, pp. 37-47, 2002.

\section{AUTHORS PROFILE}

Kohei Arai received BS, MS and PhD degrees in 1972, 1974 and 1982, respectively. He was with The Institute for Industrial Science and Technology of the University of Tokyo from April 1974 to December 1978 and also was with National Space Development Agency of Japan from January, 1979 to March, 1990. During from 1985 to 1987, he was with Canada Centre for Remote Sensing as a Post Doctoral Fellow of National Science and Engineering Research Council of Canada. He moved to Saga University as a Professor in Department of Information Science on April 1990. He was a councilor for the Aeronautics and Space related to the Technology Committee of the Ministry of Science and Technology during from 1998 to 2000. He was a councilor of Saga University for 2002 and 2003. He also was an executive councilor for the Remote Sensing Society of Japan for 2003 to 2005 . He is an Adjunct Professor of University of Arizona, USA since 1998. He also is Vice Chairman of the Commission A of ICSU/COSPAR since 2008. He wrote 29 books and published 500 journal papers.

Steven Ray Sentinuwo, received the M.Eng. degree in Information Engineering, from University of Indonesia and Dr.Eng. degree in Information Science from Saga University, in 2006 and 2013, respectively. He is currently a Post-Doctoral Fellow at Information Science in Saga University, Japan. His research interest includes robot path planning, modeling and simulation, information system management, and computer vision. 